\title{
On the Convergence of the Initial-Value Adjusting Method for Nonlinear Boundary Value Problems
}

\author{
By \\ Taketomo Mitsui*
}

\section{Introduction}

We shall consider numerical methods for solutions of a nonlinear differential equation

$$
\frac{d x}{d t}=X(t, x), \quad a<t<b
$$

subject to a general constraining condition represented by a functional of $x$

$$
f(x)=0 .
$$

Here $x$ and $X(t, x)$ are $n$-dimensional vectors, $x(t)$ is considered as a function $I=[a, b] \rightarrow \boldsymbol{R}^{n}$ and $f$ is an $n$-dimensional vector-valued functional on some subset of $C(I)$.

Recently Ojika and Kasue [5] have proposed a numerical procedure called "initial-value adjusting method" to solve the multi-point boundary value problem, which is considered to be a powerful algorithm for rather complex constraining conditions. And Ojika [4] has given a proof for convergence of their method.

The present paper is devoted to an analysis of the method by the different way from his and to obtaining sufficient conditions for convergence of the iteration. Roughly speaking, for the initial-value adjusting method, which can be regarded as a systematical version of the shooting methods, the convergence holds when $X$ and $f$ are sufficiently smooth and the starting value of iteration is taken sufficiently close to the isolated exact one. Moreover, it will be shown that the inverse of the "adjusting matrix" is a good example of the contractor that has been introduced by Altman [1].

Received October 9, 1978.

* Research Institute for Mathematical Sciences, Kyoto University. 
In the present paper, we shall denote the Euclidean norm of $n$-dimensional vector $x$ by $\|x\| . \quad C(I)$ stands for the Banach space of vector-valued continuous functions on $I$, equipped with the norm

$$
\|x\|_{C}=\max _{t \in I}\|x(t)\| \text {. }
$$

$C^{1}(I)$ means the subset of $C(I)$ of continuously differentiable functions on $I$. The norms for matrices $\boldsymbol{R}^{n} \rightarrow \boldsymbol{R}^{n}$ and other linear operators should be taken as the induced norms by vector norms.

\section{§1. The Initial-Value Adjusting Method and the Newton Iteration}

We shall consider solutions for the problem of a nonlinear differential equation

$$
\frac{d x}{d t}=X(t, x), \quad a<t<b
$$

and

$$
f(x)=0 \text {. }
$$

This supposition of (1.2) may be extremely general one which includes the multi-point boundary value condition, not to mention the initial value condition, the linear two-point boundary value condition, the periodic condition and so on. However, the supposition that will be often mentioned in the sequel, is the nonlinear multi-point boundary value condition (MPB in abbreviation): Let $f$ be an operator mapping $N$ direct product of $\boldsymbol{R}^{n}$ into $\boldsymbol{R}^{n}$

$$
f: f\left(\xi_{1}, \xi_{2}, \ldots, \xi_{N}\right), \quad \xi_{i} \in \boldsymbol{R}^{n} \quad(i=1,2, \ldots, N)
$$

and define the functional $f: C(I) \rightarrow \boldsymbol{R}^{n}$ by

$$
f(x)=f\left(x\left(t_{1}\right), x\left(t_{2}\right), \ldots, x\left(t_{N}\right)\right),
$$

where $a=t_{1}<t_{2}<\cdots<t_{N}=b$.

Let $\mathscr{D}$ be a domain of the $t x$-space bounded on $x$, intercepted by two hyperplanes $t=a$ and $t=b$. The boundary points of $\mathscr{D}$ on the hyperplanes $t=a$ and $t=b$ are supposed to be included in $\mathscr{D}$ and to make an open set on each hyperplane. Put

$$
\begin{aligned}
& D=\left\{x \in C^{1}(I) ;(t, x(t)) \in \mathscr{D} \text { for } t \in I\right\} \\
& D^{\prime}=\{x \in C(I) ;(t, x(t)) \in \mathscr{D} \text { for } t \in I\} .
\end{aligned}
$$


Then it is evident that $D \subset D^{\prime}$ and $D$ and $D^{\prime}$ are open respectively in $C^{1}(I)$ and $C(I)$.

In the problem we assume the following conditions.

Assumption 1. $X(t, x)$ is defined and twice continuously differentiable with respect to $x$ on $\mathscr{D}$, and $f(x)$ is defined and twice continuously Fréchet differentiable on $D^{\prime}$. (For MPB, $f\left(\xi_{1}, \ldots, \xi_{N}\right)$ is twice continuously differentiable with respect to each $\xi_{i}$ satisfying $\left(t_{i}, \xi_{i}\right) \in \mathscr{D}$.)

Here we shall specify some constants.

$$
\begin{aligned}
& K_{0}=\sup _{\mathscr{D}}\|X(t, x)\|, \\
& K_{1}=\sup _{\mathscr{D}}\left\|X_{x}(t, x)\right\|, \\
& C_{0}=\sup _{D^{\prime}}\|f(x)\|, \\
& C_{1}=\sup _{D^{\prime}}\left\|f_{x}(x)\right\|,
\end{aligned}
$$

where $X_{x}(t, x)$ and $f_{x}(x)$ stand for the Jacobian matrix of $X(t, x)$, the Fréchet derivative of $f(x)$ respectively.

The initial-value adjusting method for MPB proposed by Ojika-Kasue [5] can be given essentially as following:

Step 0. Choose suitable positive perturbation parameter $\varepsilon$ and initial value $\eta_{0} \in \boldsymbol{R}^{n}$, and set $k=0$.

Step 1. Compute the numerical solution $x_{k}(t)$ of (1.1) for the initial condition $x_{k}(a)=\eta_{k}$, and obtain the resulting value of the nonlinear boundary condition (1.3), i.e.

$$
f_{k}=f\left(x_{k}\right) \text {. }
$$

Step 2. If the vector $f_{k}$ is sufficiently close to 0 by the previously given criterion, terminate the iteration. Otherwise, go to the next step.

Step 3. Set $j=1$.

Step 4. Compute the numerical solution $y_{k}^{(j)}(t)$ of (1.1) for the initial condition $y_{k}^{(j)}(a)=\eta_{k}+\varepsilon e_{j}$, where $e_{j}$ is the $j$-th unit vector of $\mathbb{R}^{n}$.

Step 5. Replace $j$ by $j+1$, and return to Step 4 until $j=n$.

Step 6. Determine the matrix $S\left(\varepsilon ; x_{k}\right)$ such that

$$
\begin{aligned}
S\left(\varepsilon ; x_{k}\right)= & \left(\frac{1}{\varepsilon}\left\{f\left(y_{k}^{(1)}\right)-f_{k}\right\}, \ldots,\right. \\
& \left.\frac{1}{\varepsilon}\left\{f\left(y_{k}^{(j)}\right)-f_{k}\right\}, \ldots, \frac{1}{\varepsilon}\left\{f\left(y_{k}^{(n)}\right)-f_{k}\right\}\right) .
\end{aligned}
$$


Step 7. By the equation

$$
\eta_{k+1}=\eta_{k}-S\left(\varepsilon ; x_{k}\right)^{-1} f_{k}
$$

determine the initial value $\eta_{k+1}$ for next iteration. Then replace $k$ by $k+1$, and return to Step 1.

In the above process, the numerical integrations of the differential equation (1.1) are carried out by a suitable step-by-step method, for example, the RungeKutta method. It should be noted that, although we are considering MPB, the method can be easily extended to apply for a more general condition (1.2).

We shall call hereafter the matrix $S\left(\varepsilon ; x_{k}\right)$ the adjusting matrix (of $k$-th iteration) which plays an important role in the iteration.

On the other hand, we may construct the Newton iterative procedure for the problem of (1.1) and (1.2) (Urabe [7]). Let $F$ be an operator mapping $C^{1}(I)$ into $\mathscr{B}=C(I) \times \boldsymbol{R}^{n}$, where $\mathscr{B}$ is considered as a Banach space equipped with the norm $\left\|\left[\begin{array}{l}y \\ v\end{array}\right]\right\|_{\mathscr{B}}=\|y\|_{C}+\|v\|$,

$$
F(x)=\left[\begin{array}{l}
\frac{d x}{d t}-X(t, x) \\
f(x)
\end{array}\right]
$$

The problem of (1.1) and (1.2) has been transformed to that of finding a root for the operator equation

$$
F(x)=0
$$

in $D$. Put $J(x)$ as the Fréchet derivative of $F(x)$, then $J(x): C^{1}(I) \rightarrow \mathscr{B}$, is additive and homogeneous and

$$
J(x) y=\left[\begin{array}{l}
\frac{d y}{d t}-X_{x}(t, x) y \\
f_{x}(x) y
\end{array}\right] \text { for } y \in C^{1}(I) .
$$

As a well-known result, there exists a non-singular matrix $\Phi(t ; x)$ depending on $x$ such that

$$
\left\{\begin{array}{l}
\frac{d \Phi}{d t}=X_{x}(t, x) \Phi, \quad a<t<b, \\
\Phi(a ; x)=E_{n},
\end{array}\right.
$$

where $E_{n}$ is the unit matrix of $n$-dimension. We shall call it a matrizant for the homogeneous differential equation $\frac{d z}{d t}=X_{x}(t, x) z$. Let us define a $G$-matrix $G(x)$ by 


$$
G(x)=f_{x}(x) \Phi(\cdot ; x),
$$

then the nonsingularity of $G(x)$ yields the invertibility of $J(x)$, and vice versa.

In the case of MPB, the Fréchet derivative $f_{x}(x)$ means such operation that

$$
f_{x}(x) y=\sum_{i=1}^{N} \frac{\partial f}{\partial \xi_{i}}\left(x\left(t_{1}\right), \ldots, x\left(t_{N}\right)\right) \cdot y\left(t_{i}\right)
$$

for $y \in C(I)$. Hence the $G$-matrix is given by

$$
G(x)=\sum_{i=1}^{N} \frac{\partial f}{\partial \xi_{i}}\left(x\left(t_{1}\right), \ldots, x\left(t_{N}\right)\right) \Phi\left(t_{i} ; x\right) .
$$

Again returning back to the general case (1.8), we shall prescribe the Newton iteration. Under the assumption that $G$-matrix is nonsingular, $J(x)^{-1}$ is represented by

$$
\begin{gathered}
J(x)^{-1} \boldsymbol{b}=\Phi(t ; x) G(x)^{-1} v+\Phi(t ; x) \int_{a}^{t} \Phi^{-1}(s ; x) y(s) d s \\
-\Phi(t ; x) G(x)^{-1} f_{x}(x)\left\{\Phi(t ; x) \int_{a}^{t} \Phi^{-1}(s ; x) y(s) d s\right\}
\end{gathered}
$$

for $\boldsymbol{b}=\left[\begin{array}{l}y \\ v\end{array}\right] \in \mathscr{B}$.

The Newton iterative process:

Starting from a suitable $x_{0} \in C^{1}(I)$, generate the sequence $\left\{x_{k}\right\} \subset C^{1}(I)$ by the recurrence form

$$
x_{k+1}=x_{k}-J\left(x_{k}\right)^{-1} F\left(x_{k}\right), \quad k=0,1,2, \ldots
$$

In the iteration (1.15) let us assume that it happens that the value $\frac{d}{d t} x_{k}$ $-X\left(x_{k}, t\right)$ vanishes, then we have

$$
x_{k+1}=x_{k}-\Phi\left(t ; x_{k}\right) G\left(x_{k}\right)^{-1} f\left(x_{k}\right) .
$$

Putting $t=a$ in the above, we easily obtain the equation

$$
\begin{aligned}
x_{k+1}(a) & =x_{k}(a)-\Phi\left(a ; x_{k}\right) G\left(x_{k}\right)^{-1} f\left(x_{k}\right) \\
& =x_{k}(a)-G\left(x_{k}\right)^{-1} f\left(x_{k}\right) .
\end{aligned}
$$

Comparing (1.17) with (1.6) in the initial-value adjusting method, we shall examine the relation of two matrices $G(x)$ and $S(\varepsilon ; x)$ in the next section.

\section{§. The Nonsingularity of the Adjusting Matrix}

First, we mention a fundamental result on the continuous dependence of 
the solution on the initial value for a differential equation.

Lemma 1 ([2]). Let $\xi$ and $\eta$ be two vectors in $\boldsymbol{R}^{n}$ such that the solutions $x(t)$ and $y(t)$ for the differential equation

$$
\frac{d z}{d t}=X(t, z), \quad a<t<b
$$

subject to the initial condition

$$
z(a)=\xi \quad \text { (in the case of } x(t))
$$

or

$$
z(a)=\eta \quad(\text { in the case of } y(t))
$$

exist in D. Then we have

$$
\|x-y\|_{C} \leqq\|\xi-\eta\| e^{2 K_{1}(b-a)} .
$$

Next we state

Assumption 2. The problem (1.1) and (1.2) has an exact solution $x=\hat{x}(t)$ belonging to $D$, and the condition

$$
\operatorname{det} G(\hat{x})=\operatorname{det} f_{x}(\hat{x}) \Phi(\cdot ; \hat{x}) \neq 0
$$

is fulfilled for $\hat{x}(t)$.

Furthermore, there exists a positive constant $\bar{\varepsilon}$ such that for $0<\varepsilon \leqq \bar{\varepsilon}$ the initial value problem

$$
\left\{\begin{array}{l}
\frac{d y}{d t}=X(t, y), \quad a<t<b \\
y(a)=\hat{x}(a)+\varepsilon e_{j}
\end{array}\right.
$$

has a unique solution in $D$ for every $j$. (Throughout the paper we shall restrict $\varepsilon$ such that $0<\varepsilon \leqq \bar{\varepsilon}$.)

The first half of Assumption 2 shows the isolatedness of the solution $\hat{x}(t)$ in $D$ (Urabe [7]). Since $D$ is open in $C^{1}(I)$, for a positive constant $M_{0}$ we can take a positive number $\Delta$ such that in the $\Delta$-neighbourhood $D_{\Delta}$ of $\hat{x}$

$$
D_{\Delta}=\left\{x \in D ;\|x-\hat{x}\|_{c} \leqq \Delta\right\},
$$

$G(x)^{-1}$ exists and the inequality

$$
\left\|G(x)^{-1}\right\| \leqq M_{0}
$$

holds. We shall take and fix the number $\Delta$. Define the constant $C_{2}$ by 


$$
C_{2}=\sup _{x \in D^{\prime}} \sup _{\|\xi\|_{c} \leqq \Delta} \frac{\left\|f(x+\xi)-f(x)-f_{x}(x) \xi\right\|}{\|\xi\|_{C}^{2}} .
$$

Next we employ on the vector $X(t, x)$

Assumption 3. In $\mathscr{D}$, there exist a positive number $\delta_{0}$ and a mapping $R(\xi ; \cdot): \boldsymbol{R}^{n} \rightarrow \boldsymbol{R}^{n}$ such that the equality

$$
X(t, x+\xi)=X(t, x)+X_{x}(t, x) \xi+R(\xi ; x)
$$

holds whenever $\|\xi\| \leqq \delta_{0}$ for each $(t, x) \in \mathscr{D}$. For $R(\xi ; x)$ there exists a positive number $K_{2}$ satisfying in $\delta_{0}$-neighbourhood of $x$

$$
\|R(\xi ; x)\| \leqq K_{2}\|\xi\|^{2}
$$

uniformly to $(t, x) \in \mathscr{D}$.

Let $x(t)$ be an element of $D_{\Delta}$ satisfying

$$
\frac{d x(t)}{d t}=X[t, x(t)], \quad a<t<b .
$$

We shall analyse one iteration starting from $x(t)$. Let $\varphi^{(j)}(t ; x)$ be the $j$-th column vector of the matrizant $\Phi(t ; x)$, then $\varphi^{(j)}$ is governed by the differential equation

$$
\frac{d \varphi^{(j)}(t)}{d t}=X_{x}[t, x(t)] \varphi^{(j)}(t), \quad a<t<b
$$

subject to the initial condition

$$
\varphi^{(j)}(a ; x)=e_{j} .
$$

As is readily seen, the norm $\left\|\varphi^{(j)}\right\|_{c}$ has a bound depending on $K_{1}$, and the operator norm $\|\Phi(\cdot ; x)\|$ depends on the norm $\left\|\varphi^{(j)}\right\|_{C}$. Hence we have a positive constant $M_{1}$ such that

$$
\|\Phi(\cdot ; x)\|,\left\|\Phi^{-1}(\cdot ; x)\right\| \leqq M_{1}
$$

and

$$
\left\|\varphi^{(j)}\right\|_{C} \leqq M_{1}
$$

for $x \in D_{\Delta}$ and every $j$.

In the initial-value adjusting method we make the sequence $\left\{y^{(j)}(t)\right\}(j=1$, $2, \ldots, n)$ in $C^{1}(I)$ such that

$$
\frac{d y^{(j)}}{d t}=X\left(t, y^{(j)}\right), \quad a<t<b
$$




$$
y^{(j)}(a)=x(a)+\varepsilon e_{j} .
$$

To each $y^{(j)}$ we have

Lemma 2. $y^{(j)}(t)$ has the following expression

$$
y^{(j)}(t)=x(t)+\varepsilon\left\{\varphi^{(j)}(t)+v^{(j)}(t)\right\} \quad \text { on } \quad I,
$$

where $v^{(j)}(t)$ satisfies the differential equation

$$
\begin{aligned}
\frac{d v^{(j)}(t)}{d t}= & \frac{1}{\varepsilon}\left\{X\left[t, x(t)+\varepsilon\left\{\varphi^{(j)}(t)+v^{(j)}(t)\right\}\right]\right. \\
& -X[t, x(t)]\}-X_{x}[t, x(t)] \varphi^{(j)}(t), \quad a<t<b
\end{aligned}
$$

subject to the initial condition

$$
v^{(j)}(a)=0
$$

Proof. Easy consequence from (2.7), (2.8), (2.11), (2.12).

Since the Assumption 2 the unique existence of a solution $v^{(j)}(t)$ of (2.14) and (2.15) is evident. However we need a more precise result.

Lemma 3. For arbitrary small positive $\varepsilon$ there exists a positive constant $C^{*}$ such that the exact solution $v^{(j)}(t)$ for (2.14) and (2.15) satisfies

$$
\left\|v^{(j)}\right\|_{C} \leqq C^{*} \varepsilon \text {. }
$$

Proof. Putting $\varepsilon$ sufficiently small, we may apply Assumption 3 to the right-hand side of (2.14). Hence we have

$$
\frac{d v^{(j)}}{d t}=X_{x}[t, x(t)] v^{(j)}+\frac{1}{\varepsilon} R\left[y^{(j)}(t)-x(t) ; x\right], \quad a<t<b
$$

and

$$
v^{(j)}(a)=0
$$

By the transformation of (2.17) and (2.18) into the integral form

$$
\begin{array}{r}
v^{(j)}(t)=\int_{a}^{t} X_{x}[s, x(s)] v^{(j)}(s) d s+\frac{1}{\varepsilon} \int_{a}^{t} R\left[y^{(j)}(s)-x(s) ; x\right] d s \\
\text { for } a \leqq t \leqq b
\end{array}
$$

we obtain the following inequality:

$$
\begin{aligned}
\left\|v^{(j)}(t)\right\| \leqq \int_{a}^{t}\left\|X_{x}[s, x(s)]\right\|\left\|v^{(j)}(s)\right\| d s \\
+\frac{1}{\varepsilon} \int_{a}^{t}\left\|R\left[y^{(j)}(s)-x(s) ; x\right]\right\| d s
\end{aligned}
$$




$$
\leqq K_{1} \int_{a}^{t}\left\|v^{(j)}(s)\right\| d s+\frac{K_{2}}{\varepsilon}\left\|y^{(j)}-x\right\|_{C}^{2} \quad \text { for } \quad a \leqq t \leqq b .
$$

Applying Lemma 1 to $y^{(j)}$ and $x$ gives us

$$
\left\|y^{(j)}-x\right\|_{C} \leqq\left\|\varepsilon e_{j}\right\| e^{2 K_{1}(b-a)}=\varepsilon e^{2 K_{1}(b-a)} .
$$

Therefore the inequality

$$
\left\|v^{(j)}(t)\right\| \leqq K_{1} \int_{a}^{t}\left\|v^{(j)}(s)\right\| d s+\varepsilon K_{2} e^{4 K_{1}(b-a)} \quad \text { for } \quad a \leqq t \leqq b
$$

holds. By virtue of the Gronwall's lemma, we have the estimate

$$
\left\|v^{(j)}(t)\right\| \leqq \varepsilon K_{2} e^{5 K_{1}(b-a)} \quad \text { for } \quad a \leqq t \leqq b .
$$

Then

$$
\left\|v^{(j)}\right\|_{C} \leqq \varepsilon K_{2} e^{5 K_{1}(b-a)}
$$

holds, which gurantees the conclusion by setting $C^{*}=K_{2} e^{5 K_{1}(b-a)}$.

By virtue of the above Lemmas, we have

Theorem 1. There exists a small positive number $\varepsilon_{0}$ such that for $0<\varepsilon$ $\leqq \varepsilon_{0}$ the adjusting matrix $S(\varepsilon ; x)$ in $(1.5)$ is nonsingular while $x \in D_{\Delta}$.

Proof. Substituting (2.13) into (1.5), we have for the $j$-th column vector of $S(\varepsilon ; x)$, say $s_{j}(\varepsilon ; x)$,

$$
\begin{aligned}
s_{j}(\varepsilon ; x) & =\frac{1}{\varepsilon}\left\{f\left(y^{(j)}\right)-f(x)\right\} \\
& =\frac{1}{\varepsilon}\left\{f\left[x+\varepsilon\left\{\varphi^{(j)}+v^{(j)}\right\}\right]-f(x)\right\} \\
& =\int_{0}^{1} f_{x}\left[x+\varepsilon \theta\left\{\varphi^{(j)}+v^{(j)}\right\}\right]\left(\varphi^{(j)}+v^{(j)}\right) d \theta
\end{aligned}
$$

where $\theta$ is a variable in $0 \leqq \theta \leqq 1$. On the other hand the $j$-th column vector of $G$-matrix $G(x)$, say $g_{j}(x)$, is given by

$$
g_{j}(x)=f_{x}(x) \cdot \varphi^{(j)} .
$$

Therefore

$$
\begin{aligned}
s_{j}(\varepsilon ; x)-g_{j}(x)= & \int_{0}^{1} f_{x}\left[x+\varepsilon \theta\left\{\varphi^{(j)}+v^{(j)}\right\}\right]\left(\varphi^{(j)}+v^{(j)}\right) d \theta-f_{x}(x) \cdot \varphi^{(j)} \\
= & \int_{0}^{1}\left\{f_{x}\left[x+\varepsilon \theta\left\{\varphi^{(j)}+v^{(j)}\right\}\right]-f_{x}[x]\right\}\left(\varphi^{(j)}+v^{(j)}\right) d \theta \\
& +f_{x}(x) v^{(j)} .
\end{aligned}
$$

In Assumption 1 we supposed that $f(x)$ is twice continuously Fréchet 
differentiable on $D^{\prime}$, which implies the Lipschitz continuity of $f_{x}(x)$ on $D^{\prime}$, i.e.

$$
\left\|f_{x}(x)-f_{x}(y)\right\| \leqq 2 C_{2}\|x-y\|_{c} \quad \text { for } \quad x, y \in D^{\prime} .
$$

Besides we have had the estimates

$$
\left\|\varphi^{(j)}\right\|_{C} \leqq M_{1} \text { and }\left\|v^{(j)}\right\|_{C} \leqq C^{*} \varepsilon
$$

Hence we have

$$
\begin{aligned}
\left\|s_{j}(\varepsilon ; x)-g_{j}(x)\right\| & \leqq \int_{0}^{1}\left\|f_{x}\left[x+\theta \varepsilon\left\{\varphi^{(j)}+v^{(j)}\right\}\right]-f_{x}(x)\right\|\left\|\varphi^{(j)}+v^{(j)}\right\|_{c} d \theta \\
& \leqq \int_{0}^{1} 2 C_{2} \varepsilon \theta\left\|\varphi^{(j)}+v^{(j)}\right\|_{C}^{2} d \theta+\left\|f_{x}(x)\right\|\left\|v^{(j)}\right\|_{C} \\
& \leqq \varepsilon C_{2}\left(M_{1}+\varepsilon C^{*}\right)^{2}+\varepsilon C^{*} C_{1} .
\end{aligned}
$$

For an arbitrary small positive number $\delta_{1}$, there exists $\varepsilon_{0}$ such that for $0<\varepsilon \leqq \varepsilon_{0}$ the inequality

$$
\left\|s_{j}(\varepsilon ; x)-g_{j}(x)\right\| \leqq \frac{1}{\sqrt{n}} \delta_{1} \quad \text { for } \quad j=1,2, \ldots, n \text { and } x \in D_{\Delta}
$$

holds. Then the above inequality means

$$
\|S(\varepsilon ; x)-G(x)\| \leqq \delta_{1},
$$

which implies the nonsingularity of $S(\varepsilon ; x)$ in $D_{\Delta}$.

Corollary. For $0<\varepsilon \leqq \varepsilon_{0}$ and $x \in D_{\Delta}$, the matrix $S(\varepsilon ; x)^{-1}$ is bounded by $M_{0}\left(1-\delta_{1} M_{0}\right)^{-1}$.

\section{§3. The Convergency of the Initial-Value Adjusting Method}

In this section we shall prove the convergency of the initial-value adjusting method. Based on the idea of the method, we shall consider an operator $\mathscr{F}$ mapping $\boldsymbol{R}^{n}$ into $C^{1}(I)$, which maps the given initial value $\eta \in \boldsymbol{R}^{n}$ to a function of $C^{1}(I)$ along the flow generated by the differential equation (1.1):

$$
\begin{aligned}
& \frac{d}{d t} \mathscr{F} \eta=X[t, \mathscr{F} \eta], \quad a<t<b, \\
& \mathscr{F} \eta(a)=\eta .
\end{aligned}
$$

Throughout this section, $\varepsilon$ is restricted in the range $0<\varepsilon \leqq \varepsilon_{0}$, where $\varepsilon_{0}$ is as that mentioned in Theorem 1. As in the previous section, let us denote the exact isolated solution of the problem by $\hat{x}$ and put $\hat{\eta}=\hat{x}(a)$. The following equation is trivial: 


$$
\mathscr{F} \hat{\eta}=\hat{x}
$$

Corresponding to the iterative process of the initial-value adjusting method, an operator $\mathscr{A}$ mapping $\boldsymbol{R}^{n}$ into itself is defined by the following:

The domain of $\mathscr{A}$ denoted by $\operatorname{Dom}(\mathscr{A})$ is the totality of $\eta \in \boldsymbol{R}^{n}$ such that $(a, \eta) \in \mathscr{D}$ and $\mathscr{F} \eta \in D_{\Delta}$ hold.

$$
\mathscr{A} \eta=\eta-S(\varepsilon ; \mathscr{F} \eta)^{-1} f(\mathscr{F} \eta) \quad \text { for } \quad \eta \in \operatorname{Dom}(\mathscr{A}) \text {. }
$$

The desired solution $\hat{\eta}$ satisfies

$$
\hat{\eta}=\mathscr{A} \hat{\eta},
$$

and the iterative process is simply represented by

$$
\eta_{k+1}=\mathscr{A} \eta_{k}, \quad k=0,1,2, \ldots .
$$

For $\eta, \xi \in \operatorname{Dom}(\mathscr{A})$, the difference of $\mathscr{A} \eta$ and $\mathscr{A} \xi$ satisfies

$$
\begin{aligned}
\mathscr{A} \eta-\mathscr{A} \xi= & \eta-S(\varepsilon ; \mathscr{F} \eta)^{-1} f(\mathscr{F} \eta)-\xi+S(\varepsilon ; \mathscr{F} \xi)^{-1} f(\mathscr{F} \xi) \\
= & \eta-\xi+S(\varepsilon ; \mathscr{F} \xi)^{-1}\{S(\varepsilon ; \mathscr{F} \eta)-S(\varepsilon ; \mathscr{F} \xi)\} S(\varepsilon ; \mathscr{F} \eta)^{-1} f(\mathscr{F} \eta) \\
& -S(\varepsilon ; \mathscr{F} \xi)^{-1}\{f(\mathscr{F} \eta)-f(\mathscr{F} \xi)\} .
\end{aligned}
$$

The following lemma is well-known.

Lemma 4. The operator $\mathscr{F}$ is Fréchet differentiable in Dom $(\mathscr{A})$ and its Fréchet derivative is given by

$$
\mathscr{F}^{\prime}(\eta)=\Phi(\cdot ; \mathscr{F} \eta)
$$

for $\eta \in \operatorname{Dom}(\mathscr{A})$.

\section{Lemma 5. The estimation}

$$
S(\varepsilon ; \mathscr{F} \xi)^{-1}\{f(\mathscr{F} \eta)-f(\mathscr{F} \xi)\}=S(\varepsilon ; \mathscr{F} \xi)^{-1} G(\mathscr{F} \xi)(\eta-\xi)+o(\|\eta-\xi\|)
$$

holds.

Proof. By virtue of Lemma 4, the equation

$$
\mathscr{F} \eta-\mathscr{F} \xi=\Phi(\cdot, \mathscr{F} \xi)(\eta-\xi)+o(\|\eta-\xi\|) \text { as }\|\eta-\xi\| \rightarrow 0
$$

holds. Since

$$
f\left(x^{\prime}\right)-f(x)=f_{x}(x)\left(x^{\prime}-x\right)+O\left(\left\|x^{\prime}-x\right\|_{C}^{2}\right) \text { as }\left\|x^{\prime}-x\right\|_{C} \rightarrow 0,
$$

substitution of (3.7) yields (3.6).

In order to discuss the second term of the right-hand side of (3.4), Assumption 1 should be strengthened slightly. 
Assumption 4. In $D^{\prime}$, there exists a mapping $r(\xi ; x): D^{\prime} \rightarrow \boldsymbol{R}^{n}$ such that the equation

$$
f(x+\xi)=f(x)+f_{x}(x) \xi+\frac{1}{2} f_{x x}(x) \xi \xi+r(\xi ; x)
$$

holds whenever $\|\xi\|_{C} \leqq \delta_{0}$ for $x \in D^{\prime}$. Here $f_{x x}(x)$ means the tensor of the third order with components $\partial^{2} f_{i}(x) / \partial x_{j} \partial x_{k}(i, j, k=1,2, \ldots, n)$. For $r(\xi ; x)$ there exists a positive number $C_{3}$ satisfying in $\delta_{0}$-neighbourhood of $x$

$$
\|r(\xi ; x)\| \leqq C_{3}\|\xi\|_{C}^{3}
$$

uniformly to $x \in D^{\prime}$.

We shall take the following notations.

$$
\begin{array}{ll}
x_{1}=\mathscr{F} \eta, & y_{1}^{(j)}=\mathscr{F}\left(\eta+\varepsilon e_{j}\right), \\
x_{2}=\mathscr{F} \xi, & y_{2}^{(j)}=\mathscr{F}\left(\xi+\varepsilon e_{j}\right) .
\end{array}
$$

By virture of (2.23), we have

$$
s_{j}(\varepsilon ; \mathscr{F} \eta)=\frac{1}{\varepsilon}\left\{f\left(y_{1}^{(j)}\right)-f\left(x_{1}\right)\right\}
$$

and

$$
s_{j}(\varepsilon ; \mathscr{F} \xi)=\frac{1}{\varepsilon}\left\{f\left(y_{2}^{(j)}\right)-f\left(x_{2}\right)\right\},
$$

where $s_{j}$ stands for the $j$-th column vector of the adjusting matrix.

The equations (3.10) and (3.11) yield

$$
\begin{aligned}
& s_{j}(\varepsilon ; \mathscr{F} \eta)-s_{j}(\varepsilon ; \mathscr{F} \xi) \\
&=\frac{1}{\varepsilon}\left\{f\left(y_{1}^{(j)}\right)-f\left(x_{1}\right)-f\left(y_{2}^{(j)}\right)+f\left(x_{2}\right)\right\} \\
&=\frac{1}{\varepsilon}\left\{f\left(y_{2}^{(j)}\right)+f_{x}\left(y_{2}^{(j)}\right)\left(y_{1}^{(j)}-y_{2}^{(j)}\right)+\frac{1}{2} f_{x x}\left(y_{2}^{(j)}\right)\left(y_{1}^{(j)}-y_{2}^{(j)}\right)\right. \\
& \times\left(y_{1}^{(j)}-y_{2}^{(j)}\right)+r\left(y_{1}^{(j)}-y_{2}^{(j)} ; y_{2}^{(j)}\right) \\
&-f\left(x_{2}\right)-f_{x}\left(x_{2}\right)\left(x_{1}-x_{2}\right)-\frac{1}{2} f_{x x}\left(x_{2}\right)\left(x_{1}-x_{2}\right)\left(x_{1}-x_{2}\right) \\
&\left.-r\left(x_{1}-x_{2} ; x_{2}\right)-f\left(y_{2}^{(j)}\right)+f\left(x_{2}\right)\right\} \\
&= \frac{1}{\varepsilon}\left\{f_{x}\left(y_{2}^{(j)}\right)\left(y_{1}^{(j)}-y_{2}^{(j)}\right)-f_{x}\left(x_{2}\right)\left(x_{1}-x_{2}\right)\right. \\
&+\frac{1}{2} f_{x x}\left(y_{2}^{(j)}\right)\left(y_{1}^{(j)}-y_{2}^{(j)}\right)\left(y_{1}^{(j)}-y_{2}^{(j)}\right) \\
&-\frac{1}{2} f_{x x}\left(x_{2}\right)\left(x_{1}-x_{2}\right)\left(x_{1}-x_{2}\right)
\end{aligned}
$$




$$
\left.+r\left(y_{1}^{(j)}-y_{2}^{(j)} ; y_{2}^{(j)}\right)-r\left(x_{1}-x_{2} ; x_{2}\right)\right\} .
$$

Application of Lemma 4 brings the following two equations:

$$
\begin{aligned}
& x_{1}-x_{2}=\Phi\left(\cdot ; x_{2}\right)(\eta-\xi)+o(\|\eta-\xi\|), \\
& y_{1}^{(j)}-y_{2}^{(j)}=\Phi\left(\cdot ; y_{2}^{(j)}\right)(\eta-\xi)+o(\|\eta-\xi\|) .
\end{aligned}
$$

Thus we have the following estimations.

$$
\begin{aligned}
& f_{x}\left(y_{2}^{(j)}\right)\left(y_{1}^{(j)}-y_{2}^{(j)}\right)=f_{x}\left(y_{2}^{(j)}\right) \Phi\left(\cdot ; y_{2}^{(j)}\right)(\eta-\xi)+o(\|\eta-\xi\|), \\
& f_{x}\left(x_{2}\right)\left(x_{1}-x_{2}\right)=f_{x}\left(x_{2}\right) \Phi\left(\cdot ; x_{2}\right)(\eta-\xi)+o(\|\eta-\xi\|), \\
& f_{x x}\left(y_{2}^{(j)}\right)\left(y_{1}^{(j)}-y_{2}^{(j)}\right)\left(y_{1}^{(j)}-y_{2}^{(j)}\right)=o\left(\|\eta-\xi\|^{2}\right), \\
& f_{x x}\left(x_{2}\right)\left(x_{1}-x_{2}\right)\left(x_{1}-x_{2}\right)=o\left(\|\eta-\xi\|^{2}\right),
\end{aligned}
$$

and $r\left(y_{1}^{(j)}-y_{2}^{(j)} ; y_{2}^{(j)}\right)$ and $r\left(x_{1}-x_{2} ; x_{2}\right)$ are both $o\left(\|\eta-\xi\|^{3}\right)$.

Summing up the results, we have for the second term of the right-hand side of (3.4)

$$
\begin{aligned}
S(\varepsilon ; \mathscr{F} \xi)^{-1}\{S(\varepsilon ; \mathscr{F} \eta)-S(\varepsilon ; \mathscr{F} \xi)\} S(\varepsilon ; \mathscr{F} \eta)^{-1} f(\mathscr{F} \eta) \\
=\frac{1}{\varepsilon} S(\varepsilon ; \mathscr{F} \xi)^{-1}\left\{\Sigma(\xi)-G\left(x_{2}\right)\right\}(\eta-\xi) S(\varepsilon ; \mathscr{F} \eta)^{-1} f(\mathscr{F} \eta) \\
\quad+o(\|\xi-\eta\|),
\end{aligned}
$$

where $\Sigma(\xi)$ is a tensor of the third order whose $j$-th component is the matrix $f_{x}\left(y_{2}^{(j)}\right) \Phi\left(\cdot ; y_{2}^{(j)}\right)=G\left(y_{2}^{(j)}\right)$.

From (3.4), (3.6) and (3.15), $\mathscr{A} \eta-\mathscr{A} \xi$ is expressed by

$$
\begin{aligned}
\mathscr{A} \eta-\mathscr{A} \xi= & \left\{E_{n}-S(\varepsilon ; \mathscr{F} \xi)^{-1} G(\mathscr{F} \xi)\right\}(\eta-\xi) \\
& +\frac{1}{\varepsilon} S(\varepsilon ; \mathscr{F} \xi)^{-1}\{\Sigma(\xi)-G(\mathscr{F} \xi)\}(\eta-\xi) S(\varepsilon ; \mathscr{F} \eta)^{-1} f(\mathscr{F} \eta) \\
& +o(\|\eta-\xi\|) .
\end{aligned}
$$

Now we are ready to state

Theorem 2. Under the Assumptions 1 4, there exists a positive number $\Delta_{1}$ such that in $\Delta_{1}$-neighbourhood of $\hat{\eta}$ the initial-value adjusting iterative process converges to $\hat{\eta}$ for $0<\varepsilon \leqq \varepsilon_{0}$.

Proof. Set $\xi=\hat{\eta}$ and remember $f(\mathscr{F} \hat{\eta})=0$, then (3.16) yields

$$
\begin{aligned}
& \mathscr{A} \eta-\mathscr{A} \hat{\eta}=\left\{E_{n}-S(\varepsilon ; \mathscr{F} \hat{\eta})^{-1} G(\mathscr{F} \hat{\eta})\right\}(\eta-\hat{\eta}) \\
& \quad+\frac{1}{\varepsilon} S(\varepsilon ; \mathscr{F} \hat{\eta})^{-1}\{\Sigma(\hat{\eta})-G(\mathscr{F} \hat{\eta})\}(\eta-\hat{\eta}) S(\varepsilon ; \mathscr{F} \eta)^{-1} f(\mathscr{F} \eta) \\
& \quad+o(\|\eta-\hat{\eta}\|) \\
& =\left\{E_{n}-S(\varepsilon ; \mathscr{F} \hat{\eta})^{-1} G(\mathscr{F} \hat{\eta})\right\}(\eta-\hat{\eta})
\end{aligned}
$$




$$
\begin{aligned}
& +\frac{1}{\varepsilon} S(\varepsilon ; \mathscr{F} \hat{\eta})^{-1}\{\Sigma(\hat{\eta})-G(\mathscr{F} \hat{\eta})\}(\eta-\hat{\eta}) S(\varepsilon ; \mathscr{F} \eta)^{-1} G(\mathscr{F} \hat{\eta})(\eta-\hat{\eta}) \\
& +o(\|\eta-\hat{\eta}\|) .
\end{aligned}
$$

The first term of the right-hand side is estimated such that

$$
\begin{aligned}
\| & \left\{E_{n}-S(\varepsilon ; \mathscr{F} \hat{\eta})^{-1} G(\mathscr{F} \hat{\eta})\right\}(\eta-\hat{\eta}) \| \\
& \leqq\left\|S(\varepsilon ; \mathscr{F} \hat{\eta})^{-1}\{S(\varepsilon ; \mathscr{F} \hat{\eta})-G(\mathscr{F} \hat{\eta})\}\right\| \cdot\|\eta-\hat{\eta}\| \\
& \leqq \frac{\delta_{1} M_{0}}{1-\delta_{1} M_{0}}\|\eta-\hat{\eta}\| .
\end{aligned}
$$

Therefore $\Delta_{1}$ should be chosen so small that for $0<\varepsilon \leqq \varepsilon_{0}$ the norm of

$$
\frac{1}{\varepsilon} S(\varepsilon ; \mathscr{F} \hat{\eta})^{-1}\{\Sigma(\hat{\eta})-G(\mathscr{F} \hat{\eta})\}(\eta-\hat{\eta}) S(\varepsilon ; \mathscr{F} \eta)^{-1} G(\mathscr{F} \eta)(\eta-\hat{\eta})+o(\|\eta-\hat{\eta}\|)
$$

is smaller than $\alpha\|\eta-\hat{\eta}\|$, where $\alpha$ is a positive constant such as

$$
\alpha=\frac{1-2 \delta_{1} M_{0}}{1-\delta_{1} M_{0}}-\kappa
$$

for an arbitrary positive $\kappa<1$. This is possible because the term in question is the order $o(\|\eta-\hat{\eta}\|)$.

Then we have

$$
\begin{aligned}
\|\mathscr{A} \eta-\mathscr{A} \hat{\eta}\| & \leqq \frac{\delta_{1} M_{0}}{1-\delta_{1} M_{0}}\|\eta-\hat{\eta}\|+\left(\frac{1-2 \delta_{1} M_{0}}{1-\delta_{1} M_{0}}-\kappa\right)\|\eta-\hat{\eta}\| \\
& =(1-\kappa)\|\eta-\hat{\eta}\|,
\end{aligned}
$$

which implies the conclusion.

\section{§4. The Rate of Convergence}

As the direct consequence of (3.16) in the previous section, we have the following theorem on the Fréchet differentiability of $\mathscr{A}$.

Theorem 3. The initial-value adjusting operator $\mathscr{A}$ has the Fréchet derivative at every $\xi$ in $\operatorname{Dom}(\mathscr{A})$, which is given by

$$
\begin{aligned}
\mathscr{A}^{\prime}(\xi) \eta= & \left\{E_{n}-S(\varepsilon ; \mathscr{F} \xi)^{-1} G(\mathscr{F} \xi)\right\} \eta \\
& +\frac{1}{\varepsilon} S(\varepsilon ; \mathscr{F} \xi)^{-1}\{\Sigma(\xi)-G(\mathscr{F} \xi)\} \eta S(\varepsilon ; \mathscr{F} \xi)^{-1} f(\mathscr{F} \xi) \\
& \text { for } \eta \in \operatorname{Dom}(\mathscr{A}) .
\end{aligned}
$$

Proof is obvious.

Corollary. The Fréchet derivative $\mathscr{A}^{\prime}(\hat{\eta})$ is given by 


$$
\mathscr{A}^{\prime}(\hat{\eta})=E_{n}-S(\varepsilon ; \mathscr{F} \hat{\eta})^{-1} G(\mathscr{F} \hat{\eta})
$$

Investigation of the Fréchet derivative $\mathscr{A}^{\prime}(\hat{\eta})$ gives us the assertion that the convergence of the iteration (3.3) would not be expected quadratically in the neighbourhood of $\hat{\eta}$ while a fixed $\varepsilon$ is chosen to be apart from zero. The reason is following:

As is shown in (4.2),

$$
\begin{aligned}
\mathscr{A}^{\prime}(\hat{\eta}) \eta & =\left\{E_{n}-S(\varepsilon ; \mathscr{F} \hat{\eta})^{-1} G(\mathscr{F} \hat{\eta})\right\} \eta \\
& =S(\varepsilon ; \mathscr{F} \hat{\eta})^{-1}\{S(\varepsilon ; \mathscr{F} \hat{\eta})-G(\mathscr{F} \hat{\eta})\} \eta \\
& =S(\varepsilon ; \mathscr{F} \hat{\eta})^{-1} \sum_{j=1}^{n} \eta_{j}\left\{s_{j}(\varepsilon ; \mathscr{F} \hat{\eta})-g_{j}(\mathscr{F} \hat{\eta})\right\},
\end{aligned}
$$

where $\eta \in \operatorname{Dom}(\mathscr{A})$ and $\eta_{j}$ is the $j$-th component of vector $\eta$. By (2.23) the equation

$$
\begin{aligned}
\eta_{j} & \left\{s_{j}(\varepsilon ; \mathscr{F} \hat{\eta})-g_{j}(\mathscr{F} \hat{\eta})\right\} \\
\quad & =\eta_{j} \int_{0}^{1}\left\{f_{x}\left[\hat{x}+\varepsilon \theta\left\{\hat{\varphi}^{(j)}+\hat{v}^{(j)}\right\}\right]-f_{x}(\hat{x})\right\}\left(\hat{\varphi}^{(j)}+\hat{v}^{(j)}\right) d \theta+\eta_{j} f_{x}(\hat{x}) \hat{v}^{(j)}
\end{aligned}
$$

holds, where $\hat{x}$ stands for $\mathscr{F} \hat{\eta}$, and ${ }^{\wedge}$ means to be associated to $\hat{x}$. Since $\varepsilon$ is not zero, $f_{x}\left[\hat{x}+\varepsilon \theta\left\{\hat{\varphi}^{(j)}+\hat{v}^{(j)}\right\}\right]-f_{x}(\hat{x})$ and $\hat{v}^{(j)}$ do not vanish generally.

Hence, $\{S(\varepsilon ; \mathscr{F} \hat{\eta})-G(\mathscr{F} \hat{\eta})\} \eta$ does not always vanish for $\eta \in \operatorname{Dom}(\mathscr{A})$, which implies the spectral radius of $\mathscr{A}^{\prime}(\hat{\eta})$ is positive for a fixed $\varepsilon>0$. By virtue of the well-known theorem (e.g. Ortega-Rheinbolt [6], Krasnosel'skii et al. [3]), the positiveness of the spectral radius of $\mathscr{A}^{\prime}(\hat{\eta})$ brings the linear convergence at best.

Note that our above assertion does not deny the possibility of quadratic convergence by choosing $\varepsilon$ adaptively during the iteration. For the investigation of this direction, see Ojika [4].

Let us reformulate the problem (1.1) and (1.2) as an operator equation different from (1.7). Define an operator $\mathscr{P}: \boldsymbol{R}^{n} \rightarrow \mathbb{R}^{n}$ by

$$
\mathscr{P} \eta=f(\mathscr{F} \eta) \quad \text { for } \quad \eta \in \mathbb{R}^{n} \text {. }
$$

The domain of $\mathscr{P}$ is identical to $\operatorname{Dom}(\mathscr{A})$. The problem (1.1) and (1.2) is transformed into the form finding the root of the equation

$$
\mathscr{P} \eta=0 \text {. }
$$

It is obvious that the Fréchet derivative of $\mathscr{P}$ at $\eta$ is given by

$$
\mathscr{P}^{\prime}(\eta)=f_{x}(\mathscr{F} \eta) \Phi(\cdot ; \mathscr{F} \eta)=G(\mathscr{F} \eta)
$$


Now we can state the following result.

Theorem 4. For sufficiently small $\varepsilon, S(\varepsilon ; \mathscr{F} \eta)^{-1}$ is a contractor introduced by Altman [1].

Proof. By Example 3.1 of chapter 1 of [1], a sufficient condition for a contractor is that $S(\varepsilon ; \mathscr{F} \eta)^{-1}$ satisfies

$$
\left\|\mathscr{P}^{\prime}(\eta) S(\varepsilon ; \mathscr{F} \eta)^{-1} \eta-\eta\right\| \leqq q\|\eta\|, \quad 0<q<1 .
$$

Employing (4.5), we have

$$
\begin{aligned}
\mathscr{P}^{\prime}(\eta) S(\varepsilon ; \mathscr{F} \eta)^{-1} \eta-\eta & =G(\mathscr{F} \eta) S(\varepsilon ; \mathscr{F} \eta)^{-1} \eta-\eta \\
& =G(\mathscr{F} \eta) S(\varepsilon ; \mathscr{F} \eta)^{-1}\{G(\mathscr{F} \eta)-S(\varepsilon ; \mathscr{F} \eta)\} G(\mathscr{F} \eta)^{-1} \eta .
\end{aligned}
$$

By the same argument which we have often repeated, the right-hand side can be bounded by $q\|\eta\|$ for $0<q<1$ by choosing $\varepsilon$ sufficiently small.

Final Remark. Our discussion implicitly assumes that for any $\eta \in$ $\operatorname{Dom}(\mathscr{A}), \mathscr{F} \eta$, i.e. the solution of the differential equation $\frac{d x}{d t}=X(t, x), a<t$ $<b$ starting from the initial value $x(a)=\eta$, can be exactly computed, which does not hold strictly. Because of the nonlinearity of $X(t, x)$, the numerical integration of differential equation is necessary, and the appearance of computational error is unavoidable. However, if we can obtain a total error estimation for the employed numerical integration by some technique, we may argue the above results within the limits of the error estimation.

\section{Acknowledgment}

The present author would like to express sincere thanks to Professor Takeo Ojika, the Osaka Kyoiku University, for his helpful discussions. $\mathrm{He}$ is also grateful to Professor Tetsuro Yamamoto, Ehime University, for his suggestions to simplify the proof of Lemma 3 in the present form.

\section{References}

[1] Altman, M., Contractors and contractor directions, theory and application. Lecture Notes Pure \& Appl. Math., Marcel Dekker Inc., New York, 1977.

[2] Kimura, T., Ordinary differential equations, Kyoritsu Publ., Tokyo, 1974 (in Japanese).

[3] Красноселъский, М.А., Вайнккно, Г.М. и др., Приближенное решение операторных уравнений, Наука, Москва, 1969.

[4] Ojika, T., On quadratic convergence of the initial-value adjusting method for multi- 
point boundary-value problems, J. Math. Anal. Appl., 73 (1980), 192-203.

[5] Ojika, T. and Kasue, Y., Initial-value adjusting method for the solution of nonlinear multipoint boundary-value problems, J. Math. Anal. Appl., 69 (1979), 359-371.

[6] Ortega, J. M. and Rheinbolt, W. C., Iterative solution of nonlinear equations in several variables, Academic Press, New York, 1970.

[7] Urabe, M., The Newton method and its application to boundary value problems with nonlinear boundary conditions, Proc. U.S.-Japan Sem. Different. Funct. Eqs., Benjamin, New York, 1967, 383-410. 
